\title{
INFECCIÓN POR EL NUEVO CORONAVIRUS 2019 EN NIÑOS
}

\author{
Patricia Llaque (1D) ${ }^{1,2}$ a \\ ${ }^{1}$ Instituto Nacional de Salud del Niño San Borja, Lima, Perú. \\ ${ }^{2}$ Universidad Peruana de Ciencias Aplicadas, Lima, Perú. \\ ${ }^{a}$ Médico Pediatra Neumóloga, magíster en Ciencias de Investigación Epidemiológica.
}

\section{RESUMEN}

La enfermedad del coronavirus 2019 (COVID-19) es poco frecuente en niños y su salud se ve poco comprometida en la mayoría de casos. La presentación clínica más común es tos, fiebre y eritema faríngeo, los casos graves suelen presentarse con taquipnea. El curso de la enfermedad es de una a dos semanas. Los hallazgos de laboratorio son inespecíficos, entre ellos, linfopenia, elevación de la proteína $\mathrm{C}$ reactiva y la procalcitonina. En fases iniciales, la radiografía torácica es usualmente normal, y los hallazgos tomográficos más comunes son consolidaciones con signo del halo, vidrio esmerilado y nódulos pequeños, que afectan principalmente las zonas subpleurales. El manejo es sintomático y, en los casos graves, debe estar enfocado a brindar soporte respiratorio. Se recomienda que la manipulación de las secreciones respiratorias sea limitada y que se tengan las mismas precauciones para evitar contaminación que en pacientes adultos.

Palabras clave: Coronavirus; Enfermedad por Coronavirus 2019-nCoV; Niño (fuente: DeCS BIREME).

\section{NOVEL 2019 CORONAVIRUS INFECTION IN CHILDREN}

\section{ABSTRACT}

COVID-19 is rarely reported in children and they are mildly affected in most cases. The most common clinical presentation of COVID-19 is cough, fever and sore throat; severe cases show tachypnea. The course of the disease is from one to two weeks. Laboratory findings are nonspecific; lymphopenia, elevation of C-reactive protein and procalcitonin have been described. Early chest X-ray is usually normal, and the most common tomographic findings are consolidations with halo, ground-glass opacities and tiny nodules which mainly affects subpleural areas. Management of the disease is supportive; in severe cases, it should be focused on respiratory support. It is recommended to limit the handling of respiratory secretions and to follow the same preventive measures provided to adults

Keywords: Coronavirus Infection; 2019 Novel Coronavirus Disease; Child (source: MeSH NLM).

\section{INTRODUCCIÓN}

El 11 de marzo de 2020, la Organización Mundial de la Salud (OMS) consideró pandemia la infección por SARS-CoV-2. La enfermedad por coronavirus 2019 (COVID-19, por sus siglas en inglés) fue descrita por primera vez en diciembre de 2019, en la ciudad de Wuhan, provincia de Hubei (China). Para el 26 de abril de 2020, se habían notificado 2804796 casos y 193710 muertes en todo el mundo ${ }^{(1)}$.

Una revisión de 72314 casos de infección por SARS-CoV-2 realizada por el Centro Chino

Citar como: Llaque PB. Infección por el nuevo coronavirus 2019 en niños. Rev Peru Med Exp Salud Publica. 2020;37(2):335-40. doi: https://doi. org/10.17843/rpmesp.2020.372.5439

Correspondencia: Patricia Beatriz Llaque Quiroz, patriciallaque83@ gmail.com

Recibido: 05/04/2020 Aprobado: $22 / 04 / 2020$ En línea: 05/05/2020 para el Control y Prevención de Enfermedades encontró que solo 1\% de los casos fueron de niños y adolescentes ${ }^{(2)}$. El Centro de Control y Prevención de Enfermedades de Corea del Sur, uno de los países que ha realizado más pruebas a su población, informó hasta el 20 de marzo de 2020, que el 1,1\% y el 5,2\% fueron pacientes de cero a nueve años y de 10 a 19 años, respectivamente ${ }^{(3)}$.

En el Perú, el 5 de marzo de 2020 se confirmó el primer caso importado de COVID-19 y actualmente en los casos registrados no es posible identificar el nexo epidemiológico con algún caso confirmado ${ }^{(4)}$. Al 26 de abril del año en curso, se habían diagnosticado 27517 personas con la COVID-19 y reportado 728 fallecimientos ${ }^{(5)}$, de los cuales cuatro eran niños y uno, adolescente ${ }^{(6)}$. 
Es necesario revisar la literatura sobre la presentación de COVID-19 en niños, debido a que hay estudios preliminares que han demostrado que la frecuencia de aparición, las manifestaciones clínicas y la gravedad son diferentes a las que presentan los pacientes adultos. Además, en el contexto de una pandemia y con el poco conocimiento sobre este virus en nuestro continente, se hace necesario realizar la presente revisión narrativa cuyo objetivo es analizar la literatura científica disponible respecto a las particularidades de esta infección viral en población pediátrica.

\section{ESTRATEGIA DE BÚSQUEDA}

Se realizó una búsqueda en la base de datos PubMed hasta el 28 de marzo de 2020. Se incluyeron reportes y series de casos, estudios observacionales, ensayos clínicos y revisiones sistemáticas en niños (0-18 años) con COVID-19. La búsqueda de términos incluyó las palabras clave y los conectores: "coronavirus"[All Fields] OR (“COVID-19"[All Fields] OR “SARS-CoV-2"[All Fields]) AND ("pediatrics"[All Fields] OR ("neonates"[All Fields] OR "children"[All Fields])). Se usaron los filtros para recuperar artículos por título, resumen y texto completo.

Se encontraron 115 artículos relacionados con el tema de interés publicados entre el 1 de febrero de 2020 y el 28 de marzo de 2020. De estos, 31 fueron relevantes para esta revisión. La mayoría de los estudios fueron realizados en China.

\section{FISIOPATOLOGÍA}

Los coronavirus son un grupo de virus relacionados con la trasmisión zoonótica y causan enfermedad en mamíferos y aves. Estos virus contienen ácido ribonucléico (ARN) con una envoltura que tiene la apariencia de una corona bajo la observación microscópica debido a la presencia de glicoproteína $S$ que sobresale como espículas en su membrana y que permite la unión del virus con sus células diana ${ }^{(7)}$.

El SARS-CoV-2 es de forma redondeada, con un diámetro de $60 \mathrm{~nm}$ a $100 \mathrm{~nm}$ y pertenece al género de los $\beta$-coronavirus. El virus utiliza la enzima convertidora de angiotensina 2 (ECA2) como receptor funcional para su ingreso a la célula; esta enzima es una proteína de membrana expresada en el pulmón (neumocitos tipo 2), el corazón, el riñón y el intestino y está asociada principalmente con enfermedades cardiovasculares ${ }^{(8)}$. Luego de que el virus ingresa a la célula, el ARN viral es liberado en el citoplasma y es trasladado dentro de poliproteínas y proteínas estructurales donde ocurre su replicación, posteriormente este nuevo recubrimiento proteico que contiene las partículas virales, se fusiona con la membrana plasmática para liberar el virus de la célula ${ }^{(9)}$.

Cuando el virus ingresa a la célula, sus antígenos son presentados a las células presentadoras de antígeno (CPA), que cumplen una función importante en la inmunidad antivi- ral. Posteriormente, las CPA presentan los antígenos al complejo mayor de histocompatibilidad y luego son reconocido por los linfocitos $\mathrm{T}$ citotóxicos específicos, lo que produce la estimulación de la inmunidad celular y humoral mediada por linfocitos B y T específicos para el virus, mediante la producción de inmunoglobulinas (Ig) M y G. Los niveles de IgM se normalizan hacia la decimosegunda semana, mientras que los niveles de IgG pueden durar por largo plazo ${ }^{(9)}$.

La infección viral es capaz de producir una «tormenta de citoquinas», en consecuencia, un daño celular extenso. Esta liberación exagerada de citoquinas puede generar un síndrome de inflamación sistémica, caracterizado por fiebre y disfunción orgánica múltiple, propio de la COVID-19. La causa más común de muerte en estos pacientes es el SDRA (síndrome de dificultad respiratoria aguda). Uno de los principales mecanismos que producen el SDRA es la liberación exagerada de células inflamatorias, de citoquinas pro-inflamatorias (IFN- $\alpha$, IFN- $\gamma$, IL1b, IL-6, IL-12, IL-18, IL-33, FNT- $\alpha$, FNT- $\beta$ ) y de quimocinas (CCL2, CCL3, CCL5, CXCL8, CXCL9, CXCL10) ${ }^{(9)}$.

A nivel pulmonar, hay hallazgos característicos del daño producido por este virus, como inflamación alveolar exudativa e inflamación intersticial. Otros hallazgos patológicos encontrados son exudado fibroso, proliferación del epitelio alveolar con descamación focal, formación de las membranas hialinas con trombos en pequeños vasos y exfoliación de epitelio bronquial $^{(10)}$.

Hay tres hipótesis sobre las causas por las que la COVID-19 afecta menos a los niños. La primera sería la menor presencia de ECA2 en los pulmones de los niños ${ }^{(10)}$. La segunda es la teoría del daño endotelial: el daño endotelial previo puede facilitar e incrementar la respuesta inflamatoria al SARS-CoV-2, sin embargo, en niños sanos el daño endotelial previo está prácticamente ausente. La tercera propone que la inmunidad innata - primera línea de defensa contra el SARS-CoV-2- está estimulada en niños por la adquisición de infecciones virales comunitarias y por el esquema de vacunación ${ }^{(11,12)}$.

\section{MANIFESTACIONES CLÍNICAS}

\section{Signos y síntomas}

El Hospital de Niños de Wuhan fue el único centro destinado a la atención de niños con COVID19 en esa ciudad. Se evaluaron 1391 niños que tuvieron contacto con personas con sospecha o confirmación de la infección por SARS-CoV-2, y se estudiaron a 171 niños hospitalizados por COVID-19. Entre estos, el $60,8 \%$ fueron varones y la mediana de la edad fue 6,7 años con un rango de 1 día hasta los 15 años de edad. Con respecto a la sintomatología, el 15,8\% era asintomático; el 19,3\% tuvo síntomas del tracto respiratorio alto; y el 64,9\% tuvo neumonía.

Los síntomas más comunes fueron tos $(48,5 \%)$, eritema faríngeo $(46,2 \%)$ y fiebre por encima de $37,5{ }^{\circ} \mathrm{C}(41,5 \%)$, otros síntomas menos frecuentes fueron diarrea $(8,8 \%)$, fatiga $(7,6 \%)$, rinorrea $(7,6 \%)$ y vómitos $(6,4 \%)$. La taquipnea 
y taquicardia estuvieron presentes al ingreso al hospital en el $28,7 \%$ y el $42,1 \%$ de los pacientes, respectivamente; y solo el 2,3\% presentó saturaciones de oxígeno menor a 92\%. La mediana de la duración de la hospitalización fue de 12,5 días (rango: 1-16) ${ }^{(13)}$.

En comparación con los adultos, hay pocos casos de infección por SARS-CoV-2 en niños y se describen síntomas más leves en este grupo etario, además, un porcentaje importante de niños permanece asintomático. Sin embargo, no se descarta que la prevalencia de esta infección sea mayor en niños, por la presencia de casos asintomáticos.

\section{Gravedad}

Con respecto a la presentación de casos graves en niños, Sun D. et al. han descrito ocho casos de niños con COVID-19 cuyo diagnóstico se realizó mediante la reacción de cadena de polimerasa en muestras de hisopado nasofaríngeo, tratados en la unidad de cuidados intensivos del Hospital de Niños de Wuhan. El rango de edad fue de 2 meses a 15 años, el $75 \%$ de los afectados fueron varones. Los síntomas más frecuentes al ingreso al hospital fueron taquipnea (8/8), fiebre $(6 / 8)$ y tos (6/8). Hasta el término de este estudio, tres pacientes estuvieron bajo tratamiento en cuidados intensivos y cinco fueron dados de alta ${ }^{(14)}$. Este estudio muestra una mayor frecuencia de afectación grave en varones, hallazgo similar a lo descrito en adultos; sin embargo, la explicación de este fenómeno aún es poco clara. Todos los pacientes graves presentaron taquipnea, por lo tanto, la presencia de este signo al ingreso debe ser tomado en cuenta para decidir el manejo de niños con esta infección viral.

Sun D. et al. han descrito el aumento de la proteína C reactiva, procalcitonina y deshidrogenasa láctica en cinco de los ocho pacientes críticos, así como el aumento de alanina aminotransferasa en cuatro y dímero D en dos casos. El recuento de leucocitos, neutrófilos, linfocitos, plaquetas y hemoglobina fueron normales o estuvieron levemente afectados ${ }^{(14)}$. Sin embargo, en la evaluación con citometría de flujo, se describe que los pacientes más críticos presentan disminución del porcentaje de linfocitos CD16+ y CD56+ (4/8) y la elevación de IL-6 (2/8), IL-10 (5/8), e IFN- $\gamma$ (2/8). Además, hay aumento de linfocitos CD3+ (2/8), CD4+ (4/8) y CD8+ (1/8) ${ }^{(14)}$.

El daño pulmonar es más pronunciado en pacientes críticamente enfermos, en estos pacientes la tormenta de citoquinas se caracteriza por el aumento de las concentraciones plasmáticas de citoquinas proinflamatorias (IL-1B, IL-6, IL12 , factor de necrosis tumoral, IFN-8) y citoquinas antiinflamatorias (IL-4, IL-10, IL-13 y TGF- $\beta$ ) ${ }^{(15)}$. El aumento de linfocitos T-CD4 indica la sobreactivación del sistema inmune, lo cual puede llevar a desórdenes inmunes graves; estas alteraciones podrían conducir a daño pulmonar a largo plazo ${ }^{(14)}$.

En un estudio retrospectivo en China, en el que se evaluaron las características clínicas y epidemiológicas de 2135 niños con sospecha o confirmación de COVID-19, se des- cribió que los casos graves correspondieron al 10,6\%, 7,3\%, $4,2 \%, 4,1 \%$ y $3 \%$ en menores de un año, de uno a cinco, de seis a diez, de 11 a 15 y de 16 a 18 años, respectivamente. Estos hallazgos sugieren que los lactantes son más propensos a presentar casos graves y críticos. Esta serie también muestra que solo un paciente varón de 14 años falleció ${ }^{(16)}$.

\section{Contagiosidad}

En un estudio descriptivo con diez niños hospitalizados, el periodo de incubación promedio fue de 6,5 días, en un rango de 2 a 10 días, y la diseminación del virus en las muestras tomadas de las vías respiratorias fue prolongada, lo cual impone un reto al control de infecciones. A diferencia de los adultos, en este estudio no se encontró viremia y en el 83,3\% de los pacientes se detectó el virus en las heces por más de dos semanas y hasta un mes de duración ${ }^{(17)}$.

En los estadios tempranos de la epidemia, se ha descrito un patrón de rápido aumento del número de casos en niños. Luego se ha observado una disminución gradual y estable, en esta etapa la mayoría de los casos fueron niños tratados por COVID-19, con historia conocida de exposición a otros miembros de la familia y a otros niños con la enfermedad (16). Estos datos sugieren trasmisión familiar, conocimiento que sustenta el reforzamiento de las medidas de higiene para disminuir la propagación de la infección.

\section{Neonatos}

Se han publicado estudios que han evaluado las muestras de líquido amniótico, cordón umbilical y leche de madres afectadas por COVID-19, las cuales tuvieron un resultado negativo para SARS-CoV-2 ${ }^{(18,19)}$. Sin embargo, un estudio de cohorte que evaluó 33 recién nacidos de madres con COVID-19, informó sobre tres neonatos con la enfermedad. En cuatro de los 33 neonatos el síntoma más común fue dificultad respiratoria. Los hallazgos radiográficos fueron inespecíficos y no se notificaron muertes. Los síntomas fueron leves y el desenlace clínico, favorable. El caso más grave pudo deberse a la prematuridad, asfixia y sepsis, más que a la infección por SARS-CoV-2. Esta evidencia no es concluyente para excluir la transmisión vertical del virus, por lo que son importantes tomar medidas, como el tamizaje materno, el control de infecciones, la cuarentena de las madres y el monitoreo cercano de síntomas en los recién nacidos ${ }^{(20)}$.

\section{DIAGNÓSTICO}

\section{Reacción de cadena de polimerasa}

La reacción en cadena de la polimerasa con transcriptasa inversa en tiempo real (rt-PCR, por sus siglas en inglés) permite el diagnóstico etiológico de la COVID-19 mediante la detección del ARN del SARS-CoV-2 en hisopados nasofaríngeos y orofaríngeos ${ }^{(21)}$. Una alternativa diagnóstica es el 
secuenciamiento genético del SARS-CoV-2 en muestras del tracto respiratorio o sangre ${ }^{(22)}$.

\section{Exámenes de laboratorio}

En una revisión de 12 artículos, que incluían información sobre 66 pacientes pediátricos, se describen alteraciones en los índices leucocitarios. El 69,6\% de los casos tuvo un recuento normal de leucocitos, en el 15,2\% de casos estuvieron aumentados y en otro $15,2 \%$ disminuidos. La mayoría de los pacientes tuvo recuentos normales de neutrófilos; el 4,6\%, valores superiores, y el $6 \%$, inferiores al rango normal ${ }^{(21)}$. Por el contrario, en adultos el aumento de leucocitos y neutrófilos fue común en pacientes con progresión desfavorable ${ }^{(23)}$.

Solamente el 3\% de los niños tuvieron linfopenia. En adultos, la linfopenia se asocia a un pronóstico desalentador ${ }^{(24)}$. La proteína C reactiva estuvo elevada en el 13,6\% de niños y la procalcitonina en el 10\%. En adultos, estos valores son elevados en casos de progresión desfavorable. En niños, el aumento de la procalcitonina estuvo asociado a sobreinfección bacteriana ${ }^{(21)}$. Otro hallazgo de las pruebas de laboratorio en niños fue el incremento de las transaminasas y de la creatinina-fosfocinasa ${ }^{(25)}$. La diferencia en los hallazgos de laboratorio con respecto a los adultos puede deberse a variaciones en la respuesta inmune ante la infección viral.

En niños, se han observado incrementos significativos de los niveles de la IL-6 y de la proteína C reactiva, en los primeros cinco días de la hospitalización ${ }^{(16)}$. Se ha informado una elevación de niveles de IL-6 en otras infecciones virales en niños, y los niveles altos de IL-6 se asocian a un aumento de la mortalidad en niños menores de 5 años con neumonía grave que requieren ventilación mecánica ${ }^{(26)}$. El incremento de los niveles de esta interleucina ha sido descrito como desencadenante de la tormenta inflamatoria que caracteriza la COVID-19 ${ }^{(9)}$.

\section{Diagnóstico por imágenes}

En la radiografía torácica de los niños no hay hallazgos anormales en las fases tempranas de la enfermedad. La tomografía computada muestra cuatro fases según el patrón de afectación imagenológica. En el estadio temprano, las lesiones en su mayoría son bilaterales con hiperdensidades subpleurales y consolidación con signo del halo; este patrón se observó en 10 de 20 pacientes; también se observaron imágenes con patrón en vidrio esmerilado en 12 pacientes y nódulos pequeños solo en 3 pacientes.

En los estadios avanzados, se observa que la lesión se expande y la densidad aumenta comprometiendo múltiples lóbulos en ambos pulmones. Las imágenes en patrón de vidrio esmerilado e imágenes de consolidación coexisten con el engrosamiento septal interlobular, lesiones de fibrosis y broncograma aéreo.
En el estadio crítico, se observa que hay lesiones de consolidación pulmonar difusa y que los pulmones están comprometidos, se muestra como un "pulmón blanco» con broncograma aéreo. En los estadios de recuperación las consolidaciones se tornaron en patrón de vidrio esmerilado y luego se redujeron gradualmente hasta imágenes que revelan algunas bandas fibrosas ${ }^{(25)}$.

Los hallazgos descritos indican que los patrones en la tomografía computada de tórax causadas por el SARS-CoV-2 son compatibles con las descritas para otros patógenos virales, bacterianos y fúngicos; por lo que la evaluación epidemiológica y etiológica deben ser complementarias. Además, en comparación con los adultos, se observa mayor compromiso de regiones subpleurales.

\section{Diagnóstico diferencial}

El diagnóstico diferencial incluye infecciones por influenza, parainfluenza, adenovirus, virus sincitial respiratorio, rinovirus, metapneumovirus; así como Chlamydia pneumonia, Mycoplasma pneumoniae y neumonía bacteriana ${ }^{(27)}$.

\section{MANEJO TERAPÉUTICO}

En la revisión no se identificaron ensayos clínicos de medicamentos que se estén realizando específicamente en niños. Algunos autores recomiendan la terapia antiviral, que ya se ha usado en casos graves, pero no hay datos de su eficacia en niños.

\section{Antiinflamatorios}

Se ha sugerido que el uso de ibuprofeno puede aumentar los niveles de ECA2 expresados en células epiteliales del pulmón, intestino, riñón y vasos sanguíneos, y a través de este mecanismo, el ibuprofeno puede favorecer la unión del SARS-CoV-2 con sus células blanco ${ }^{(28)}$. Sin embargo, no hay evidencia suficiente para dejar de usar esta medicación en el tratamiento de la fiebre o el dolor en este grupo de pacientes.

\section{Hidroxicloroquina}

Se ha realizado un ensayo clínico no aleatorizado para evaluar la eficacia de la hidroxicloroquina en la eliminación viral y curso clínico de la COVID-19. En dicho ensayo uno de los criterios de inclusión fue tener más de 12 años de edad, sin embargo, el promedio de la edad de los participantes fue de 45,1 años con desviación estándar de 22 años, por lo cual los resultados no muestran datos específicos para menores de 18 años ${ }^{(29)}$.

\section{Terapia de soporte}

Según la investigación de Sun D. et al. el manejo de los niños críticamente enfermos se centró en el soporte sintomático y 
respiratorio con oxígeno a alto flujo y ventilación mecánica; además se describieron otros tratamientos como el uso de antivirales, antibióticos, corticoides e inmunoglobulinas ${ }^{(14)}$.

\section{Antivirales}

Se ha informado sobre el uso de antivirales (rivabirina, oseltamivir e interferón) en los pacientes críticos ${ }^{(14)}$. Sin embargo, no hay evidencia actual de su utilidad. Se han evaluado otros fármacos como lopinavir y ritonavir, pero sin incluir en este estudio a niños. El uso de lopinavir-ritonavir en pacientes adultos graves no mostró beneficio en la mejoría clínica, mortalidad a los 28 días, ni en la eliminación viral ${ }^{(30)}$.

\section{Antibióticos}

El uso de antibióticos debe realizarse cuando se sospeche de sobreinfección bacteriana. En caso de presentar sepsis, debería ser tratada según las guías internacionales para su manejo ${ }^{(31)}$.

\section{Corticoides}

En un estudio observacional con 31 pacientes adultos con COVID-19 leve que recibieron corticoides, el uso de esta medicación no se asoció con el tiempo de la eliminación del virus, la estancia hospitalaria o la duración de los síntomas ${ }^{(32)}$. Sin embargo, el uso de metilprednisolona redujo el riesgo de muerte en pacientes adultos con COVID-19 grave que desarrollaron SDRA ${ }^{(33)}$.

\section{Tocilizumab}

Las personas con curso fatal de COVID-19 presentan niveles elevados de IL-6 en sangre, por lo cual se ha estudiado el uso de anticuerpos monoclonales específicos que bloquean la IL-6 en casos graves. En un estudio retrospectivo con 20 casos graves de COVID-19 en adultos, el uso de tocilizumab se asoció a la disminución del requerimiento de oxígeno, a la reducción de fiebre y opacidades pulmonares, y a la recuperación del porcentaje de linfocitos en la sangre periférica ${ }^{(34)}$. Sin embargo, sus costos, disponibilidad y efectos adversos pueden ser una barrera para su uso en otras poblaciones.

\section{Plasma inmune}

El plasma inmune o convaleciente es aquel extraído de individuos luego de la resolución de la infección y del desarrollo de anticuerpos contra el virus. La administración pasiva de anticuerpos a través de la transfusión de plasma convaleciente puede ser una estrategia como profilaxis posexposición o tratamiento de enfermos. Se postulan algunos mecanismos para su efecto, los anticuerpos se unen al patógeno, lo neutralizan y pueden activar el sistema de complemento, la fagocitosis y la citotoxicidad celular dependiente de anticuerpos. El plasma convaleciente tiene un efecto a corto plazo y no excluye los riesgos propios de la transfusión. Se ha reportado su eficacia para tratar otros virus; sin embargo, aún se están llevando a cabo ensayos clínicos para evaluar su eficacia en COVID-19 ${ }^{(35)}$.

\section{Manejo de secreciones y prevención de transmisión}

La aspiración y toma de muestra de secreciones respiratorias (incluidos el esputo inducido y el lavado broncoalveolar) deben estar limitadas. Si se realizan procedimientos que involucren la vía aérea de estos pacientes, se deben tener las mismas precauciones de contaminación que los pacientes adultos con esta enfermedad ${ }^{(31)}$.

El virus es altamente contagioso y puede ser trasmitido por una persona infectada o por un portador asintomático. Las gotas respiratorias son su principal ruta de trasmisión, pero también puede ser trasmitido por contacto de las mucosas orales, nasales u oculares con el virus desde superficies contaminadas, como las manos. El tracto digestivo puede ser otra forma de infección, mediante la ingesta de restos fecales que pueden contener el virus ${ }^{(36)}$, sin embargo, la importancia este último mecanismo requiere ser establecida en estudios posteriores.

El riesgo de transmisión de la infección vía gotas y aerosoles aumenta durante los tratamientos con nebulización debido al potencial para generar un volumen alto de aerosoles respiratorios que pueden ser eliminados a distancia mayor que el patrón de dispersión natural. El uso de nebulizadores en esta pandemia tiene el potencial de trasmitir SARS-CoV-2 viables a huéspedes susceptibles, por lo que debería ser limitado a enfermedades pulmonares crónicas como fibrosis quística o pacientes que no colaboran o que no han mostrado mejoría con el uso de inhaladores de dosis medida ${ }^{(37)}$.

\section{Criterios de alta}

El consenso de expertos chinos recomienda dar el alta hospitalaria cuando se cumplan tres criterios: la temperatura corporal es normal por tres días, los síntomas respiratorios han mejorado y la prueba rt-PCR para SARS-CoV-2 es negativa en dos oportunidades y con un intervalo mínimo de un día entre ellas ${ }^{(27)}$.

\section{CONCLUSIONES}

Los niños desarrollan la enfermedad producida por el SARS-CoV-2 de manera infrecuente y con cuadro clínico leve, la radiografía es normal en fases iniciales. El manejo es fundamentalmente sintomático y con soporte respiratorio. Deben tenerse las precauciones para evitar contagios con la frecuencia que se han dado entre adultos.

Contribución de autores: LPB concibió el manuscrito, revisó la literatura, redactó y aprobó la versión final del manuscrito.

Financiamiento: Autofinanciado.

Conflicto de interés: Ninguno. 


\section{REFERENCIAS BIBLIOGRÁFICAS}

1. World Health Organization. Coronavirus disease 2019 (COVID-19), situation reports [Internet]. [citado el 26 de abril del 2020]. Disponible en: https://www.who.int/docs/default-source/coronaviruse/situation-reports/20200426-sitrep-97-covid-19.pdf?sfvrsn=d1c3e800_6.

2. Wu Z, McGoogan JM. Characteristics of and important lessons from the coronavirus disease 2019 (COVID-19) outbreak in China: summary of a report of 72,314 cases from Chinese Center for Disease Control and Prevention. JAMA. 2020. doi: 10.1001/jama.2020.2648.

3. Korean Center for Disease and Control and Prevention [Internet]. Press Releases. [citado el 24 de marzo del 2020]. Disponible en: https://www. cdc.go.kr/board/board.es?mid=a30402000000\&bid=0030.

4. Ministerio de Salud. Vigilancia, Prevención y Control del COVID-19. Alerta epidemiológica 13 [Internet]. Lima: Dirección General de Epidemiología, MINSA; 2020 [citado el 27 de marzo de 2020]. Disponible en: https://www.dge.gob.pe/portal/docs/alertas/2020/AE013.pdf.

5. Ministerio de salud. Comunicado No78 [Internet]. Lima, MINSA; 2020. [citado el 26 de abril del 2020]. Disponible en: https://www.gob.pe/ institucion/minsa/noticias/131667-minsa-casos-confirmados-por-coronavirus-covid-19-ascienden-a-27-517-en-el-peru-comunicado-n-78.

6. Ministerio de salud [Internet]. Lima, MINSA;2020. [citado el 26 de abril del 2020]. Disponible en: https://covid19.minsa.gob.pe/sala_situacional.asp.

7. Zhu N, Zhang D, Wang W, Li X, Yang B, Song J, et al. Coronavirus Investigating, and Research Team. A novel coronavirus from patients with pneumonia in China, 2019. N Engl J Med. 2020; 382(8):727-733. doi: 10.1056/NEJMoa2001017.

8. Jin Y, Yan H, Ji W, Wu w, Chen S, Zhang W, et al. Virology, Epidemiology, Pathogenesis, and Control of COVID-19. Viruses. 2020; 12(4). pii: E372. doi: 10.3390/v12040372.

9. Li X, Geng M, Peng Y, Meng L, Lu S. Molecular immune pathogenesis and diagnosis of COVID-19. J. Pharm. Anal. 2020; 10(2): 102-108 doi: https://doi.org/10.1016/j.jpha.2020.03.001.

10. Jain A. COVID-19 and lung pathology. Indian J Pathol Microbiol. 2020; 63:171-2.

11. Lee PI, Hu YL, Chen PY, Huang YC, Hsueh PR. Are children less susceptible to COVID-19? J Microbiol Immunol Infect. 2020. pii: S16841182(20)30039-6. doi: 10.1016/j.jmii.2020.02.011.

12. García-Salido A. Three Hypotheses About Children COVID19. The Pediatric Infectious Disease Journal. 2020. doi: 10.1097/INF.0000000000002701

13. Lu X, Zhang L, Li YY, Liu D, Shen K, Xu S, et al. SARS-CoV-2 Infection in Children. N Engl J Med 2020; 382:1663-1665. doi: 10.1056/ NEJMc2005073

14. Sun, D., Li, H., Lu, X. et al. Clinical features of severe pediatric patients with coronavirus disease 2019 in Wuhan: a single center's observational study. World J Pediatr. 2020. doi: 10.1007/s12519-020-00354-4.

15. Huang C, Wang Y, Li X, Ren L, Zhao J, Hu Y, et al. Clinical features of patients infected with 2019 novel coronavirus in Wuhan, China. Lancet. 2020; 395:497-506. doi: 10.1016/S0140-6736(20)30183-5.

16. Dong Y, Mo X, Hu Y, Qi X, Jiang F, Jiang Z, et al. Epidemiology of COVID-19 Among Children in China. Pediatrics. 2020; 145(6):1-10. doi: https://dx.doi.org/10.1542/peds.2020-0702.

17. Cai J, Xu J, Lin D, Yang Z, Xu L, Qu Z, et al. A Case Series of children with 2019 novel coronavirus infection: clinical and epidemiological features. Clin Infect Dis 2020; 28:28. doi: https://dx.doi.org/10.1093/cid/ciaa198.

18. Zhu H, Wang L, Fang C, Peng S, Zhang L, Chang G, et al. Clinical analysis of 10 neonates born to mothers with 2019-nCoV pneumonia. Transl Pediatr. 2020;9(1):51-60. doi: 10.21037/tp.2020.02.06.

19. Chen H, Guo J, Wang C, Luo F, Xuechen Y, Zhang W, et al. Clinical characteristics and intrauterine vertical transmission potential of COVID-19 infection in nine pregnant women: a retrospective review of medical records. Lancet. 2020;395(10226):809-815. doi: 10.1016/S0140-6736(20)30360-3.

20. Zeng L, Xia S, Yuan W, Yan K, Xiao F, Shao J, et al. Neonatal Early-Onset
Infection With SARS-CoV-2 in 33 Neonates Born to Mothers With COVID-19 in Wuhan, China. JAMA Pediatrics. 2020. doi: 10.1001/ jamapediatrics.2020.0878.

21. Henry BM, Lippi G, Plebani M. Laboratory abnormalities in children with novel coronavirusdisease 2019. Clin Chem Lab Med 2020; 16:16. doi: https://doi.org/10.1515/cclm-2020-0272.

22. Ludvigsson JF. Systematic review of COVID-19 in children show milder cases and a better prognosis than adults. Acta Paediatrica. 2020; 00:1-8. doi: 10.1111/apa.15270.

23. Lippi G, Plebani M. Laboratory abnormalities in patients with COVID-2019 infection. Clin Chem Lab Med 2020. doi: 10.1515/cclm2020-0198.

24. Chen N, Zhou M, Dong X, Qu J, Gong F, Han Y, et al. Epidemiological and clinical characteristics of 99 cases of 2019 novel coronavirus pneumonia in Wuhan, China: a descriptive study. Lancet 2020;395:507-13. doi: https:// doi.org/10.1016/S0140-6736(20)30211-7.

25. Xia W, Shao J, Guo Y, Xehua P, Zhen L, Hu D. Clinical and CT features in pediatric patients with COVID-19 infection: Different points from adults. Pediatr Pulmonol. 2020; 55(5):1169-1174. doi: 10.1002/ppul.24718.

26. Nguyen Thi Dieu T, Pham Nhat A, Craig TJ, Duong-Quy S. Clinical characteristics and cytokine changes in children with pneumonia requiring mechanical ventilation. J Int Med Res 2017; 45:1805-17. doi: https://doi. org/10.1177/0300060516672766.

27. Shen K, Yang Y, Wang T, Zhao D, Jiang Y, Jin R, et al. Diagnosis, treatment, and prevention of 2019 novel coronavirus infection in children: experts' consensus statement. World J Pediatr 2020; 07:07. doi: https://dx.doi. org/10.1007/s12519-020-00343-7.

28. Fang L, Karakiulakis G, Roth M. Are patients with hypertension and diabetes mellitus at increased risk for COVID-19 infection? Lancet Respir Med. 2020; 8(4):e21. doi: 10.1016/S2213-2600(20)30116-8.

29. Gautret P, Lagier JC, Parola P, Hoang VT, Meddeb L, Mailhe M, et al. Hydroxichloroquine and azithromycin as a treatment of COVID-19: results of an open-label non-randomized clinical trial. Int J Antimicrob Agents. 2020: 105949. doi: 10.1016/j.ijantimicag.2020.105949.

30. Cao B, Wang Y, Wen D, Liu W, Wang J, Fan G, et al. A trial of Lopinavir-Ritonavir in Adults Hospitalized with Severe Covid-19. N Engl J Med. 2020. doi: 10.1056/NEJMoa2001282.

31. Sociedad Española de Pediatría [Internet]. Documento de manejo clínico del paciente pediátrico con infección por SARS-CoV-2. [Citado el 23 de marzo del 2020]. Disponible en: https://www.seipweb. es/wp-content/uploads/2020/03/13_marzo_documento_aep-seipscip-seup-corregido.pdf.

32. Zha L, LiS, Pan L, Tefsen B, Li Y, French N, et al. Impact of corticosteroid treatment in patients with coronavirus disease 2019. Med J Aust. 2020. doi: $10.5694 / \mathrm{mja} 2.50577$.

33. Wu C, Chen X, Cai Y, Xia J, Zhou X, Xu S, et al. Risk factors associated with acute respiratory distress syndrome and death in patients with coronavirus disease 2019 pneumonia in Wuhan, China. JAMA Intern Med. 2020. doi: 10.1001/jamainternmed.2020.0994.

34. Xu XL, Han MF, Li TT, Sun W, Wang DS, Fu BQ, et al. Effective treatment of severe COVID-19 patients with tocilizumab. ChinaXiv:202003.00026.

35. Bloch EM, Shoman S, Casadevall A, Sachais BS, Shaz B, Winters JL, et al. Deployment of convalescent plasma for the prevention and treatment of COVID-19. J Clin Invest. 2020. pii: 138745. doi: 10.1172/JCI138745.

36. Riou J, Althaus CL. Pattern of early human-to-human transmission of Wuhan 2019 novel coronavirus (2019-nCov). December 2019 to January 2020. Euro Surveill. 2020; 25:7-11. doi: https://doi.org/10.2807/15607917.ES.2020.25.4.2000058.

37. Amirav I, Newhouse MT. Transmission of Corona Virus by Nebulizer - a serious, underappreciated risk. CMAJ. 2020;192(7):171-172. doi: https:// doi.org/10.1503/cmaj.75066. 